\title{
STROMGREN TRAILS OF HOT WHITE DHARPS
}

\author{
John C. Raymond \\ Harvard-Smithsonian Center for Astrophysics
}

\section{ABSTRACT}

High resolution IUE spectra of Feige 24 reveal sharp C IV absorption features at both the stellar velocily and the velocity of the interstellar 1ines. Dupree and Raymond have shown that the ionization zone created by Feige 24 itself accounts for the interstellar components of C IV and Si IV, and that the density of hot white dwarfs in the galaxy implies that similar ionized zones around hot white dwarfs make a significant contribution to the $C$ IV column densities observed toward distant $O$ and $B$ stars. Typical white dwarf velocities of around $30 \mathrm{~km} / \mathrm{s}$ and C IV ionization zone diameters less than 1 pc imply that the crossing time is comparable to the ionization time, so that non-equilibrium ionization models are required. Models of the resulting elongated trails are presented.

\section{MODELS}

Most techniques for studying interstellar gas average along the line of sight. 0 stars and supernovae light up specific regions for study, but they tend to occur in abnormal regions of space, and they strongly perturb the 11luminated gas. By measuring the $\mathrm{C} I V, \mathrm{Si} I V$ and $\mathrm{N} V$ column densities associated with a hot white dwarf, we can determine the ambient density of the gas near the white dwarf. Space Telescope may make it possible to observe enough hot white dwarfs to determine the filling factor of the warm component of the ISM in the neighborhood of the Sun.

The major difficulty in such a determination in confusion of narrow stellar absorption lines of C IV, SI IV and N V (e.g. Sion, Guinan and Wesemael 1982; Bruhweller and Kondo 1981) with interstellar lines. Feige 24 is a convenient object for study in that the stellar features are redshifted and their velocity varies with the 4.2 day orbital period, while the interstellar features stay fixed near zero velocity (Dupree and Raymond 1982). While the interstellar C IV and Si IV might be unassociated with Feige 24, these lines are not present in spectra of a star $1^{\circ}$ from Felge 24 but only 20 pe away or of a more distant star $3^{\circ}$ from Feige 24. The column densities of C IV and Si IV and the upper limit on $N$ V agree with predictions of a simple static $H$ II region model provided that the density near Feige 24 is at least $0.1 \mathrm{~cm}^{-3}$ (Dupree and Raymond 1983). The model of a static photoionized region is only approximate, however, since typical white dwarf velocities $(30 \mathrm{~km} / \mathrm{s}$ ) are large enough that the ionization time is comparable to the time in which the white dwarf crosses the zone of high ionization. Thus non-equilibrium ionization models are required.

A computer code designed to model radiative interstellar shock waves 
(Raymond 1979) has been modified to compute the structure of the ionized trail left by a hot white dwarf moving through the interstellar gas. Many improvements to the atomic rates in the code have also been made, of which the relevant ones are Reilman and Manson (1979) photoionization cross sections, low temperature dielectronic recombination (Nussbaumer and Storey 1983) and charge transfer (as described in Butler and Raymond 1980). Figure 1 shows the 1\%, 3\%, $10 \%$ and $30 \%$ contours of the C IV, Si IV and N V ionization fractions for a model specific to Feige 24. A radius of $0.2 R_{0}$, a temperature of 70,000 $\mathrm{K}$ and a velocity of $60 \mathrm{~km} / \mathrm{s}$ were used. The stellar flux distribution was taken from the $\log g=8$ pure hydrogen model of Wesemael et al (1980). The density was held constant at $0.1 \mathrm{~cm}^{-3}$, and a background ionization rate sufficient to maintain $95 \%$ ionization of hydrogen and $5000 \mathrm{~K}$ was assumed to approxImate the 'warm ionized' component of the interstellar medium. Figure 2 shows the 3\% contours of C IV for different assumed density, white dwarf radius, and ambient ionization state. The models having neutral and ionized ambient gas differ in that the neutral gas is heated to $20,000 \mathrm{~K}$ when it is ionized, while the ionized ambient gas is heated only to about $8,000 \mathrm{~K}$. Thus if the ambient gas is neutral, the heating will result in expansion, and the high pressure will drive a weak shock ahead of the white dwarf.

The predicted column density can vary by an order of magnitude depending on whether the white dwarf is approaching or receding. Since Feige 24 is moving away at $34^{\circ}$ to the line of sight, its interstellar lines are unusually strong. Column densities for the model shown in Figure 1 and solar abundances are listed in Table 1.

This work has been supported by NASA contract NAG 5-87 to the Smithsonian Astrophysical Observatory.

\section{REFERENCES}

Bruhweiler, F.C., and Kondo, Y. 1981, Ap. J., 248, L123.

Butler, S., and Raymond, J.C. 1980, Ap. J., 240, 680.

Dupree, A.K., and Raymond, J.C. 1982, Ap. J., 263, L63.

Dupree, A.K., and Raymond, J.C. 1983, Ap. J., 275, L71.

Nussbaumer, H., and Storey, P.J. 1983, Astr. Ap. Suppl., in press.

Raymond, J.C. 1979, Ap. J. Suppl., 39, 1.

Re1lman, R.F., and Manson, S.T. 1979, Ap. J. Suppl., 40, 819.

Sion, E.M., Guinan, E.F., and Wesemael, F. 1982, Ap. J., 255, 232.

Wesemael, F., Auer, L.H., Van Horn, H.M., and Savedoff, M.P. 1980, Ap. J. Suppl., 228, 127.

TABLE 1

\begin{tabular}{lcccc}
\hline ion & approaching & perpendicular & $34^{\circ}$ & receding \\
C IV & 13.08 & 13.26 & 13.50 & 13.59 \\
Si IV & 12.30 & 12.43 & 12.72 & 13.22 \\
N V & 12.46 & 12.62 & 12.95 & 13.64 \\
\hline
\end{tabular}




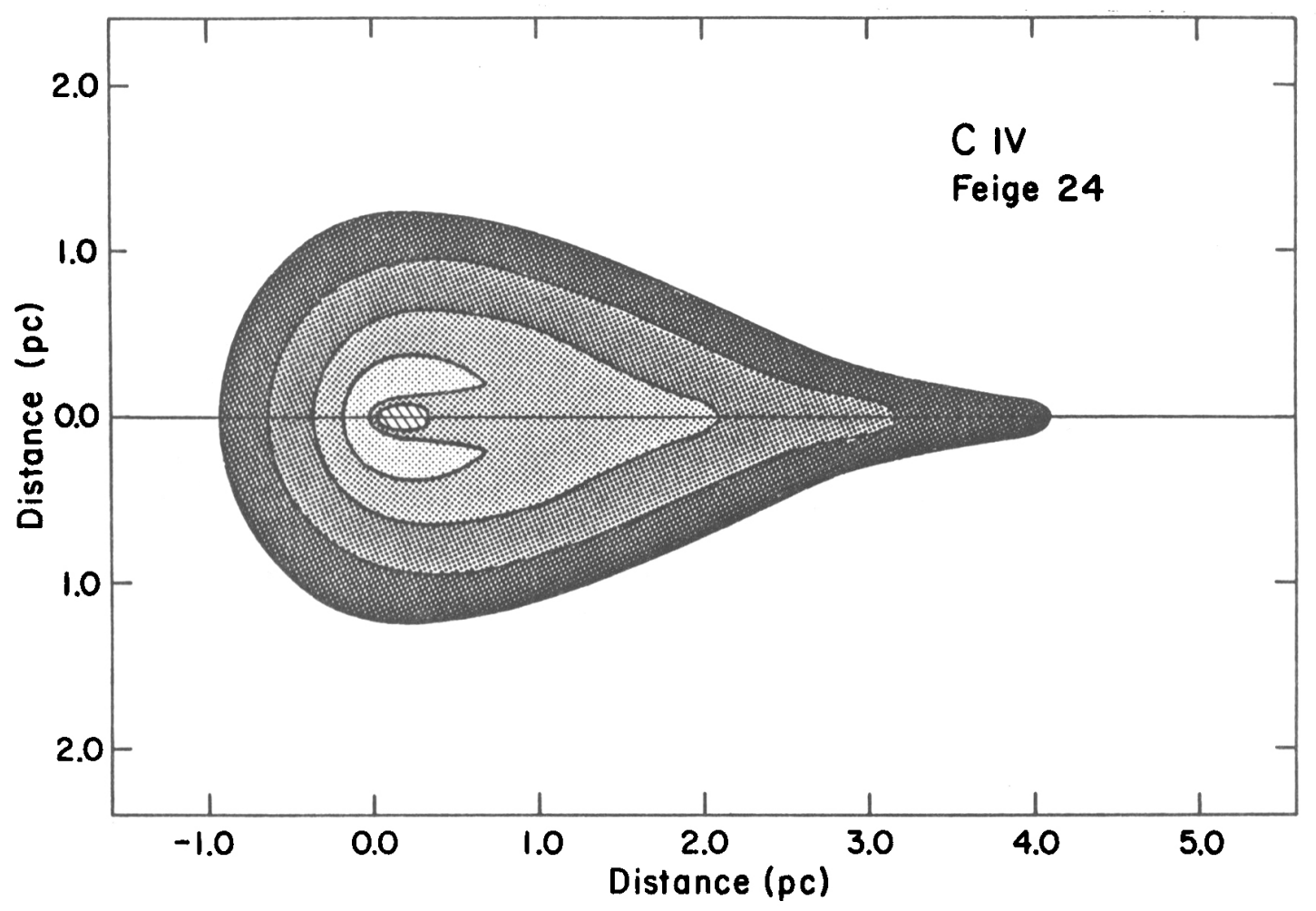

Figure la.

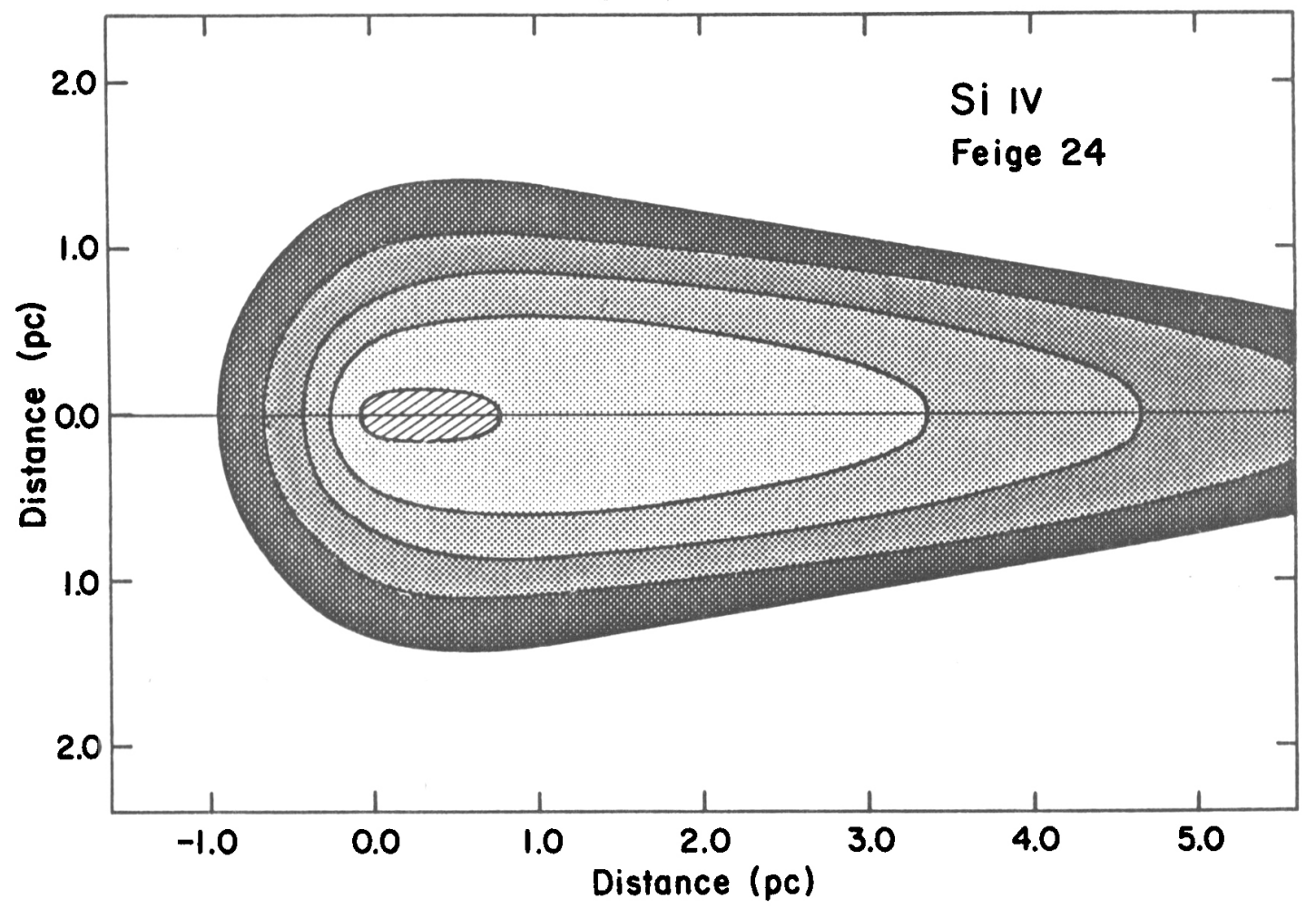

Figure 1b. 


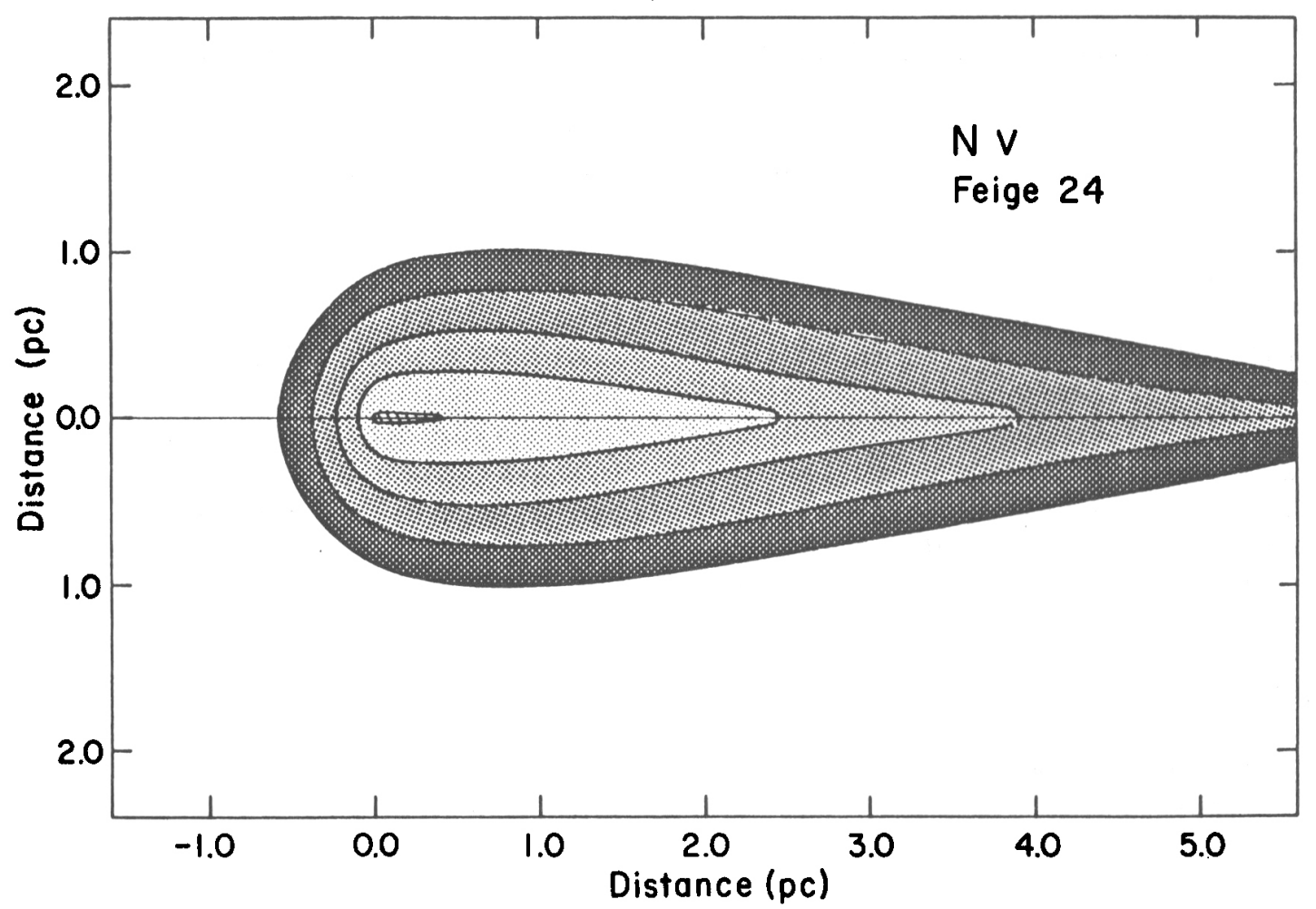

EIgure 1c.

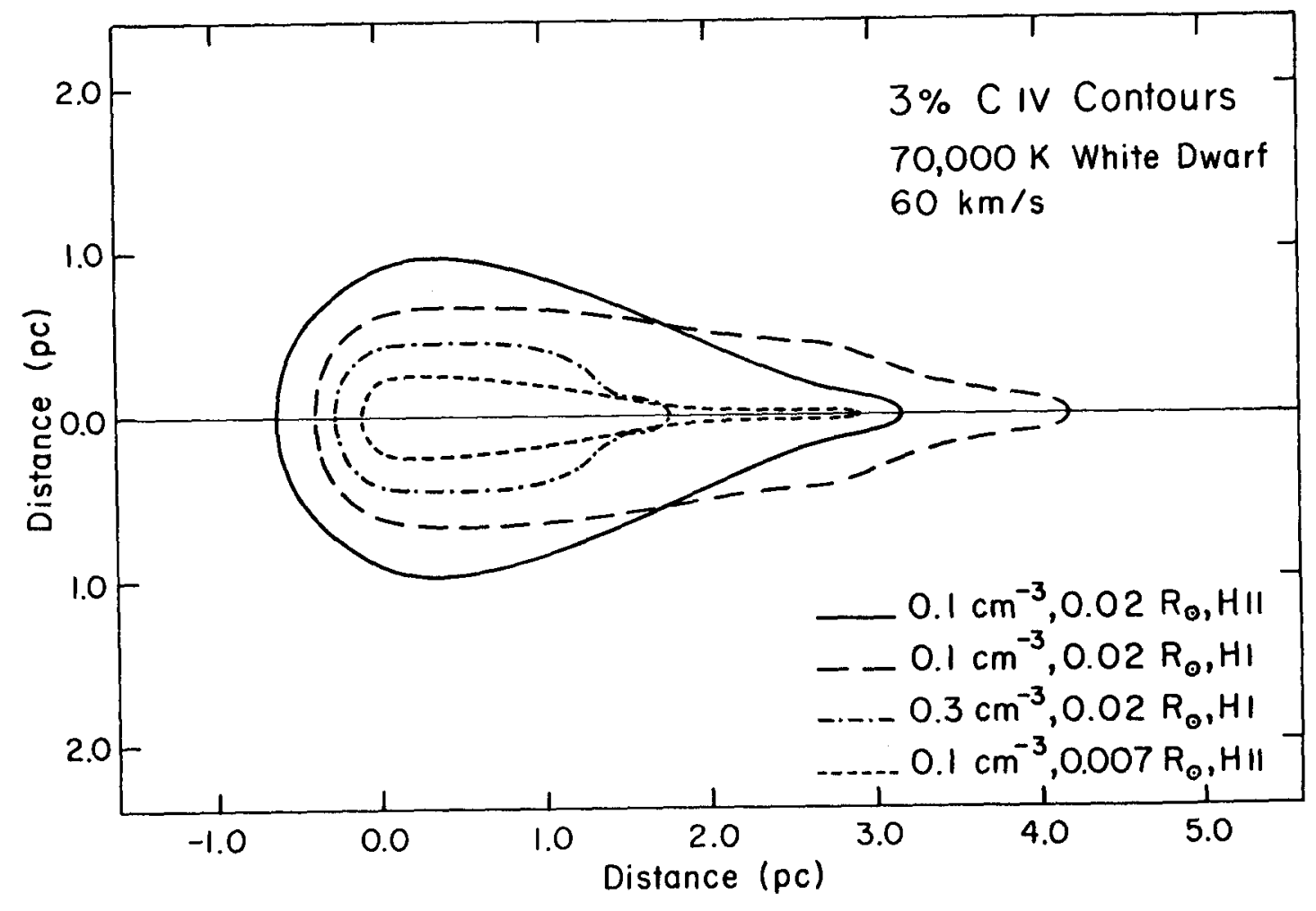

Figure 2 . 